\title{
Development and experimental verification of a genome-scale metabolic model for Corynebacterium glutamicum Yohei Shinfuku $^{\dagger 1}$, Natee Sorpitiporn ${ }^{\dagger 1}$, Masahiro Sono, Chikara Furusawa*1,2, Takashi Hirasawa ${ }^{1}$ and Hiroshi Shimizu*1
}

\begin{abstract}
Address: ${ }^{1}$ Department of Bioinformatic Engineering, Graduate School of Information Science and Technology, Osaka University, 1-5 Yamadaoka, Suita, Osaka 565-0871, Japan and 2ERATO Complex Systems Biology Project, JST, 1-5 Yamadaoka, Suita, Osaka 565-0871, Japan

Email: Yohei Shinfuku - y-shinfuku@ist.osaka-u.ac.jp; Natee Sorpitiporn - n-sorpitiporn@ist.osaka-u.ac.jp; Masahiro Sono - m-sono@ist.osakau.ac.jp; Chikara Furusawa* - furusawa@ist.osaka-u.ac.jp; Takashi Hirasawa - hirasawa@ist.osaka-u.ac.jp; Hiroshi Shimizu* - shimizu@ist.osakau.ac.jp

* Corresponding authors †Equal contributors
\end{abstract}

Published: 3 August 2009

Microbial Cell Factories 2009, 8:43 doi:10.1 186//475-2859-8-43
Received: 22 April 2009

Accepted: 3 August 2009

This article is available from: http://www.microbialcellfactories.com/content/8/1/43

(c) 2009 Shinfuku et al; licensee BioMed Central Ltd.

This is an Open Access article distributed under the terms of the Creative Commons Attribution License (http://creativecommons.org/licenses/by/2.0), which permits unrestricted use, distribution, and reproduction in any medium, provided the original work is properly cited.

\begin{abstract}
Background: In silico genome-scale metabolic models enable the analysis of the characteristics of metabolic systems of organisms. In this study, we reconstructed a genome-scale metabolic model of Corynebacterium glutamicum on the basis of genome sequence annotation and physiological data. The metabolic characteristics were analyzed using flux balance analysis (FBA), and the results of FBA were validated using data from culture experiments performed at different oxygen uptake rates.
\end{abstract}

Results: The reconstructed genome-scale metabolic model of C. glutamicum contains 502 reactions and 423 metabolites. We collected the reactions and biomass components from the database and literatures, and made the model available for the flux balance analysis by filling gaps in the reaction networks and removing inadequate loop reactions. Using the framework of FBA and our genome-scale metabolic model, we first simulated the changes in the metabolic flux profiles that occur on changing the oxygen uptake rate. The predicted production yields of carbon dioxide and organic acids agreed well with the experimental data. The metabolic profiles of amino acid production phases were also investigated. A comprehensive gene deletion study was performed in which the effects of gene deletions on metabolic fluxes were simulated; this helped in the identification of several genes whose deletion resulted in an improvement in organic acid production.

Conclusion: The genome-scale metabolic model provides useful information for the evaluation of the metabolic capabilities and prediction of the metabolic characteristics of $C$. glutamicum. This can form a basis for the in silico design of $C$. glutamicum metabolic networks for improved bioproduction of desirable metabolites.

\section{Background}

A coryneform bacterium, Corynebacterium glutamicum is a facultatively aerobic, gram-positive bacterium that can grow on various sugars or organic acids $[1,2]$. This organism can produce various amino acids such as glutamate $[1,2]$ and lysine [3] with high efficiency, and is thus widely 
used for the large-scale production of amino acids [4,5]. Furthermore, the production of ethanol and organic acids such as lactate and succinate by growing C. glutamicum under oxygen deprivation conditions has recently been proposed $[6,7]$. Owing to its importance for bioproduction, C. glutamicum has been chosen as one of the effective hosts for metabolic engineering purposes [8-10]. Thus, the construction and exploration of appropriate in silico metabolic models, which help predict the cellular behavior and production of useful chemicals, are highly desired.

Recently, on the basis of whole-genome information, the genome-scale metabolic networks of cells have been reconstructed and applied to metabolic flux balance analysis (FBA) $[11,12]$ for many organisms, including representatives of each of the 3 major domains of life, namely, archaea [13], bacteria [14-17], and eukarya [18-20]. FBA is an analysis of metabolic flux profiles, in which a steady state of metabolic flux is assumed and the profile of metabolic fluxes is calculated by optimizing an objective function using linear programming. Although genome-scale metabolic models cannot compute the detailed kinetic dynamics of metabolic reactions in a cell, they enable the description of the range of possible metabolic states on the basis of the constraints defined by the stoichiometry of metabolic reactions and transport steps at a steady state. Furthermore, we can obtain a solution, i.e., a set of all metabolic fluxes, which maximizes an objective function using linear programming. The biomass production rate is generally adopted as the objective function. It has been shown that the metabolic profiles calculated by the maximization of biomass production can describe those obtained experimentally in many organisms and environmental conditions, suggesting that organisms can maximize their growth rate by adaptation and evolution $[21,22]$. Using the appropriate genome-scale metabolic network and an objective function to be maximized, FBA can be used to predict the relationship among the genotype, environmental conditions, and product yields at the steady state; this data can be utilized for the improvement of the microbial production $[23,24]$.

In this study, we present the reconstruction of a genomescale metabolic model of C. glutamicum. Metabolic reactions and other parameters for biomass were collected using databases and literatures. After reconstruction of the genome-scale metabolic model, we performed FBA simulations with the maximization of biomass production and evaluated the results of the simulations by using experimental data of C. glutamicum cultures grown at different oxygen uptake rates (OURs). Our results revealed that the production rates of biomass and organic acids predicted using our model agreed well with the experimental rates; this result suggests that this model well represents the intracellular metabolic profiles of C. glutamicum. It should be noted that the representation of such changes in the metabolic profiles that occur on changing the oxygen uptake is difficult for another genome-scale model of $C$. glutamicum reported recently [25]. Furthermore, by using the model proposed in this paper, we performed comprehensive simulations of gene deletions in order to identify candidate genes for genetic modification(s) to improve the productivity of organic acids by C. glutamicum.

\section{Materials and methods \\ Modeling and Simulations}

Network reconstruction

The known metabolic reactions in the C. glutamicum metabolic network were collected by a search of public databases and scientific publications. The genome-scale metabolic network was based on the pathways in the BioCyc database collection [26] http://www.biocyc.org for $C$. glutamicum. We also referred to the information on $C$. glutamicum in the Kyoto Encyclopedia of Genes and Genomes database (KEGG; http://www.kegg.jp). In general, the genome-scale model constructed using only public databases contains incorrect and insufficient metabolic pathways owing to the incompleteness of database information. The most frequently observed incorrectness was missing enzymes in metabolic pathways. Thus, the resulting network was then subjected to the gap-filling process to allow biomass formation. For gap filling, we referred to published cell-specific data available in the literatures, such as Ref. $[27,28]$ and references threin. For example, the mycolic acid and arabinogalactan synthetic pathways are constructed on the basis of Refs. [29,30].

\section{Determination of biomass composition}

The reaction "biomass synthesis" is a hypothetical metabolite in the metabolic network, which represents the requirement of precursors and coenzymes for the biomass formation. Biomass synthesis consists of a linear combination of 43 components, including amino acids, DNA, RNA, lipids, and cell envelope components. In our model, the biomass composition was determined from various reported data. The demands of precursors such as pyruvate, acetyl-CoA, and oxaloacetate for biomass production were based on the data in Ref. [27]. For the macromolecular composition, we referred to the data in Ref. [31]; the total biomass is composed of $52 \%$ protein, $5 \%$ RNA, $1 \%$ DNA, $13 \%$ lipid, $19 \%$ cell wall components, and $10 \%$ other components. The C. glutamicum cells have a characteristic cell membrane termed as MAPc [32], which consists of the polysaccharides peptidoglycan and arabinogalactan as well as mycolic acids. Since the MAPc biosynthesis pathway is quite complex and some metabolic reactions in MAPc synthesis have not been characterized in detail, we described this pathway by some lumped reactions. The biomass composition of MAPc was determined to satisfy the macromolecular composition and the 
precursor demand described above. The biomass compositions of nucleic acids were calculated on the basis of the genomic sequence [33]; the composition ratios of DNA and RNA to the total biomass are $5 \%$ and $1 \%$, respectively [31]. The biomass synthesis reactions and its composition are shown in additional file 1. For all simulations presented here, the composition of every component was fixed independent of the environmental conditions. The energy requirement for biomass production was set to $41.26 \mathrm{mmol}$ of ATP per $1 \mathrm{~g}$ biomass on the basis of the data in Ref.[34].

\section{In silico computation: FBA}

Metabolic fluxes of the C. glutamicum metabolic network were calculated by using flux balance analysis (FBA), in which constrains are imposed by the stoichiometry in a metabolic network [11]. A pseudo-steady state is generally assumed, i.e., the net sum of all production and consumption fluxes for each internal metabolite is set to zero. This assumption results in a feasible space that is a convex set in the N-dimensional space of metabolic fluxes (where $\mathrm{N}$ stands for the total number of fluxes). In FBA, a particular objective function, written as a linear combination of fluxes, can be used to calculate the optimal solution at 1 corner in the feasible flux space. Using the matrix notation, this problem can be stated as follows:

$$
\begin{gathered}
\text { maximize : } \boldsymbol{c}^{\mathrm{T}} \cdot \boldsymbol{v} \\
\text { subject to }: \boldsymbol{S} \cdot \boldsymbol{v}=0 \\
\boldsymbol{v}_{\min } \leq \boldsymbol{v} \leq \boldsymbol{v}_{\max },
\end{gathered}
$$

where $\mathbf{S}$ is the stoichiometric matrix representing the stoichiometry of metabolic reactions in the network and $v$ is a vector of all metabolic fluxes. $\boldsymbol{v}_{\min }$ and $\boldsymbol{v}_{\max }$ indicate the minimum and maximum constraints on the fluxes and are used to define the constraints for maximal enzymatic rate, irreversibility of reaction, or constant uptake from the environment. $c^{\mathrm{T}}$ is a vector representing the objective function to be maximized, as a linear combination of metabolic fluxes. In general, the biomass production rate mentioned above is used as the objective function to be maximized. In this study, we followed this method to calculate the metabolic flux profile under the assumption that organisms have been evolved toward growth maximization. For all simulations in this paper, glucose was chosen as the sole carbon source and the following external metabolites were allowed to freely transport through the cell membrane: $\mathrm{CO}_{2}, \mathrm{H}_{2} \mathrm{O}, \mathrm{SO}_{3}, \mathrm{NH}_{3}$, and $\mathrm{PO}_{4}$. All calculations, including the linear programming problems, were performed using the commercially available software Lindo (Lindo Systems, Inc.) and Matlab (Mathworks, Inc.).

\section{Strain and medium}

C. glutamicum strain ATCC 13032 was used in the culture experiments. The composition of the synthetic medium used for the preculture of the microorganism was the same as that employed in our previous study [35] (per liter of deionized water): $40 \mathrm{~g}$ of glucose, $30 \mathrm{~g}$ of $\left(\mathrm{NH}_{4}\right)_{2} \mathrm{SO}_{4}, 3.0 \mathrm{~g}$ of $\mathrm{Na}_{2} \mathrm{HPO}_{4}, 6.0 \mathrm{~g}$ of $\mathrm{KH}_{2} \mathrm{PO}_{4}, 2.0 \mathrm{~g}$ of $\mathrm{NaCl}, 84 \mathrm{mg}$ of $\mathrm{CaCl}_{2}, 3.9 \mathrm{mg}$ of $\mathrm{FeCl}_{3}, 0.9 \mathrm{mg}$ of $\mathrm{ZnSO}_{4} .7 \mathrm{H}_{2} \mathrm{O}, 0.1 \mathrm{mg}$ of $\left(\mathrm{NH}_{4}\right)_{6} \mathrm{Mo}_{7} \mathrm{O}_{21} \cdot 4 \mathrm{H}_{2} \mathrm{O}, 0.3 \mathrm{mg}$ of $\mathrm{Na}_{2} \mathrm{~B}_{4} \mathrm{O}_{7} .10 \mathrm{H}_{2} \mathrm{O}, 0.4 \mathrm{mg}$ of $\mathrm{MgSO}_{4} .7 \mathrm{H}_{2} \mathrm{O}, 40 \mathrm{mg}$ of $\mathrm{FeSO}_{4} \cdot 7 \mathrm{H}_{2} \mathrm{O}, 500 \mu \mathrm{g}$ of vitamin $\mathrm{B} 1 \cdot \mathrm{HCl}, 0.1 \mathrm{~g}$ of EDTA, and $10 \mu \mathrm{g}$ of biotin. The medium composition for the main culture was the same as that for the preculture except the initial glucose concentration was changed to $80 \mathrm{~g} / \mathrm{L}$ for batch cultivation and $20 \mathrm{~g} / \mathrm{L}$ for continuous cultivation.

\section{Culture conditions}

For microaerobic culture conditions (experiments 1 and 2 shown in Fig. 1(a)), the batch cultivations of C. glutamicum were carried out using a 500-mL jar fermenter (model BMJ-P, Able, Japan) with a liquid working volume of 200 $\mathrm{mL}$. In the batch cultures, the cells were first allowed to grow aerobically at a high aeration rate ( $1 \mathrm{vvm}$; volume of air per volume of medium per min) and high agitation speed $(800 \mathrm{rpm})$ until the cell concentration, as measured by the optical density at $660 \mathrm{~nm}\left(\mathrm{OD}_{660}\right)$, reached $5 \sim 15$. Then, for experiment 1 , the culture conditions were changed to no aeration and gentle agitation (100 rpm) and for experiment 2, the aeration rate was altered to 0.5 vvm and the OUR was maintained at a constant value $(0.5$ $\mathrm{mmol} / \mathrm{gDW} / \mathrm{h}$ ) by changing the agitation speed. For the aerobic cultures (experiments 3, 4, and 5), chemostat cultivations were carried out using a 500-mL jar fermenter with a liquid working volume of $200 \mathrm{~mL}$. The aeration rate was fixed at $1 \mathrm{vvm}$ and the dilution rate, at $0.2 \mathrm{~h}^{-1}$ for all chemostat culture experiments. In order to change the OUR in these chemostat cultures, the agitation speed was set to 800,1000 , and $1100 \mathrm{rpm}$ for experiments 3, 4, and 5 , respectively.

All culture experiments were performed at $31.5^{\circ} \mathrm{C}$, and the $\mathrm{pH}$ was maintained at 7.2 by the automatic addition of $25 \%$ ammonium solution using an autoclavable $\mathrm{pH}$ probe (Fermprobe F-635; Broadley-James Corporation, Irvine, CA) and a pH controller (DT-1023; Able Corporation, Japan).

\section{Analytical Methods}

Cell growth was monitored by measuring the $\mathrm{OD}_{660}$. The dry cell weight (DCW) was calculated using the measured $\mathrm{OD}_{660}$ according to the following formula:

$$
\operatorname{DCW}(\mathrm{g} / \mathrm{L})=0.30 \times \mathrm{OD}_{660} \text {. }
$$


(a)

\begin{tabular}{|c|c|c|c|c|c|c|c|c|c|c|c|}
\hline experiment & $\begin{array}{l}\text { calture } \\
\text { method }\end{array}$ & $\begin{array}{c}\text { agitation } \\
(\mathrm{rpm}) \\
\end{array}$ & GUR & OUR & OUR/GUR & & $\mathrm{CO}_{2}$ & Lactate & Acetate & Succinate & Biomass \\
\hline 1 & batch & 100 & 1.48 & 0 & 0 & $\begin{array}{l}\exp \\
\ldots \ldots \\
\text { sim }\end{array}$ & $\begin{array}{l}\mathrm{ND} \\
(-) \\
(0 \%) \\
(0 \%)\end{array}$ & $\begin{array}{c}1.93 \\
(65.2 \%) \\
(68.5 \%)\end{array}$ & $\begin{array}{c}0.08 \\
(1.7 \%) \\
(0.8 \%) \\
(0.8 \%)\end{array}$ & \begin{tabular}{c}
0.19 \\
$(8.7 \%)$ \\
\hdashline$(12.8 \%)$
\end{tabular} & $\begin{array}{c}0.024 \\
(10.3 \%) \\
(17.8 \%)\end{array}$ \\
\hline 2 & batch & $200-400$ & 2.68 & 0.57 & 0.21 & $\begin{array}{l}\exp \\
\ldots \ldots \\
\operatorname{sim}\end{array}$ & $\begin{array}{c}0.53 \\
(3.3 \%) \\
(3.1 \%) \\
(3 \%\end{array}$ & $\begin{array}{c}2.34 \\
(43.4 \%) \\
(52.0 \%)\end{array}$ & $\begin{array}{c}0.21 \\
(2.6 \%) \\
(1.0 \%)\end{array}$ & $\begin{array}{c}0.13 \\
(3.1 \%) \\
(19.2 \%)\end{array}$ & $\begin{array}{c}0.101 \\
(23.5 \%) \\
(24.5 \%)\end{array}$ \\
\hline 3 & chemostat & 800 & 2.06 & 3.29 & 1.59 & $\begin{array}{l}\exp \\
\ldots \ldots \\
\operatorname{sim}\end{array}$ & $\begin{array}{c}2.92 \\
(23.6 \%) \\
(31.4 \%)\end{array}$ & $\begin{array}{c}0.64 \\
(3.4 \%) \\
(0 \%)\end{array}$ & $\begin{array}{c}0.63 \\
(11.7 \%) \\
(10.4 \%)\end{array}$ & $\begin{array}{l}0.392 \\
(1.3 \%) \\
(0 \%)\end{array}$ & $\begin{array}{c}0.197 \\
(59.57 \%) \\
(58.1 \%)\end{array}$ \\
\hline 4 & chemostat & 1000 & 2.33 & 5.50 & 2.36 & $\begin{array}{l}\exp \\
\ldots \ldots \\
\operatorname{sim}\end{array}$ & $\begin{array}{c}5.48 \\
(39.2 \%) \\
(38.0) \\
\end{array}$ & $\begin{array}{c}1 \times 10^{-2} \\
(0.2 \%) \\
(0 \%)\end{array}$ & $\begin{array}{c}3 \times 10^{-3} \\
(0.1 \%) \\
(0 \%)\end{array}$ & $\begin{array}{l}\mathrm{ND} \\
(-) \\
(0 \%)\end{array}$ & $\begin{array}{c}0.197 \\
(52.8 \%) \\
(62.0 \%)\end{array}$ \\
\hline 5 & chemostat & 1100 & 2.34 & 6.61 & 2.82 & $\begin{array}{l}\exp \\
\cdots \ldots \\
\text { sim }\end{array}$ & $\begin{array}{c}6.13 \\
(47.7 \%) \\
(38.0) \\
\end{array}$ & $\begin{array}{c}1 \times 10^{-2} \\
(0.3 \%) \\
(0 \%)\end{array}$ & $\begin{array}{c}6 \times 10^{-3} \\
(0.1 \%) \\
(0 \%)\end{array}$ & $\begin{array}{l}\text { ND } \\
(-) \\
(0 \%)\end{array}$ & $\begin{array}{c}0.197 \\
(52.6 \%) \\
(62.0 \%)\end{array}$ \\
\hline
\end{tabular}

(b)

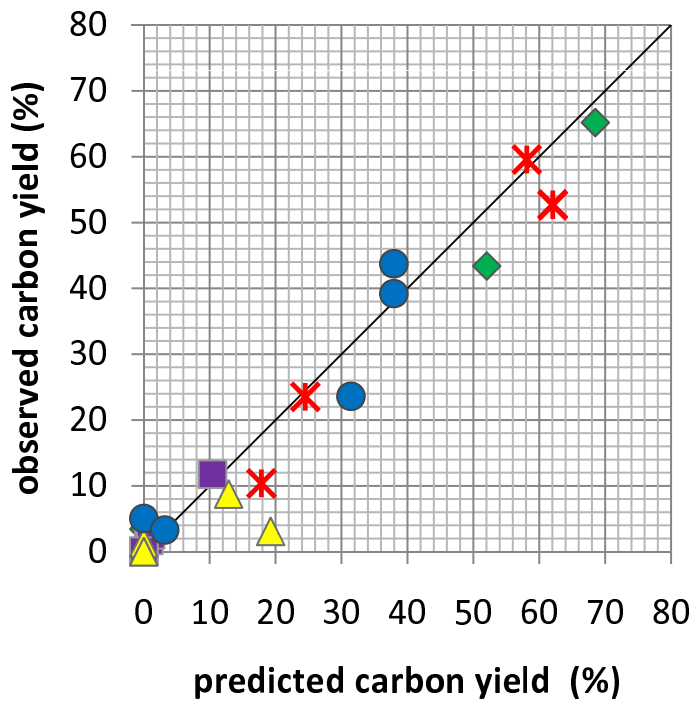

\author{
Lactate \\ Acetate \\ $\triangle$ Succinate \\ - $\mathrm{CO} 2$
}

* Biomass

Figure I

Changes in the yields of organic acids, biomass, and carbon dioxide on changing OUR/GUR ratio. (a) Summary of experimental results and predictions by FBA simulations. The unit of GUR, OUR, and production rates of $\mathrm{CO}_{2}$, lactate, acetate, succinate, and biomass are $\mathrm{mmol} / \mathrm{gDW} / \mathrm{h}$. The values in parentheses represent carbon yields. Simulation results which were obtained by using the same GUR and OUR are also presented. (b) A scatter plot of carbon yield. The x-axis corresponds to the result of FBA simulation, while the $y$-axis show the experimentally observed carbon yield. The carbon yields in the 5 sets of experimental and simulation results are presented. The line corresponding to $y=x$ is also included.

The culture supernatant was used for measuring the concentration of glucose and organic acids. Glucose was measured using an enzymatic assay kit, the Glucose CII test Wako (Wako Pure Chemicals, Inc., Japan) according to the manufacturer's protocol. Organic acids were quantified using a HPLC system, Hitachi L-6200 equipped with a L-4000H UV detector (210 nm; HITACHI, Japan). Samples were eluted with $0.75 \mathrm{mM} \mathrm{H}_{2} \mathrm{SO}_{4}$ in an ion-exclusion column TSKgel Oapak-P (Tosho, Japan) at $40^{\circ} \mathrm{C}$. The flow rate was set to $0.8 \mathrm{ml} / \mathrm{min}$. The concentration of dissolved oxygen (DO) in the culture was monitored by using an oxygen electrode (DKK-Toa Corporation, Japan), and the concentrations of exhaust oxygen and carbon dioxide were monitored by using an exhaust gas analyzer (Model DEX-1562-1; Able Corporation, Japan).

\section{Results and discussions}

\section{Reconstruction of the metabolic network}

We reconstructed a genome-scale metabolic network for C. glutamicum ATCC 13032, whose genomic DNA sequence was determined by 2 independent research groups [33,36]; the metabolic network consists of 277 
genes, 502 metabolic reactions, and 423 metabolites. The entire reaction data set is provided in additional file 1. A total of 428 reactions from the BioCyc and KEGG database collections were included in the model, while the remaining 74 reactions were added on the basis of previously published studies of each pathway $[27,28]$. The basic characteristics of the reconstructed metabolic network are presented in Table 1, in which the genomic features of C. glutamicum were obtained from Ref.[33]. From the entire set of reactions, 470 correspond to intracellular reactions, while 32 are fluxes for transport through the membrane. The model includes 391 intracellular metabolites and 32 extracellular metabolites. The functional classification of the 502 metabolic reactions in the reconstructed model is summarized in Table 2, in which the reaction "Biomass synthesis" indicates the hypothetical reaction to synthesize biomass. Transport processes were added to the model by reference to the BioCyc database collection and transport classification database (TCDB; http://www.tcdb.org) and by inference from physiological considerations and genome annotations [27].

The reconstructed metabolic network of C. glutamicum has several characteristics that distinguish it from the networks of other microorganisms. The cell envelopes of coryneform bacteria and mycobacteria have a unique structure consisting of a covalently linked complex comprising mycolic acid, arabinogalactan, and peptidoglycan (MAPc) [32]. In order to represent the characteristics of cell envelope biosynthesis, we introduced metabolic reactions for MAPc biosynthesis into the model. The synthetic pathways and composition of MAPc were decided on the basis of previous studies $[29,30]$ and the demand of MAPc for biomass production was considered to be consistent

Table I: Genomic features and characteristics of a reconstructed metabolic model of $C$. glutamicum ATCC 13032.

\begin{tabular}{lc}
\hline Feature & Property \\
\hline Genome characteristics & 3282708 bp \\
Genome length & $53.80 \%$ \\
G+C content & 3432 \\
No. of open reading frames (ORFs) & 3002 \\
Total coding sequences (CDS) & 2489 \\
CDS encoding annotated proteins & \\
& \\
In silico metabolic networks & 277 \\
No. of genes included & 428 \\
No. of associated reactions & 74 \\
No. of other reactions & 423 \\
No. of metabolites & 470 \\
No. of internal fluxes & 32 \\
No. of exchange fluxes &
\end{tabular}

The genomic features of $C$. glutamicum ATCC 13032 shown here were reported by Kalinowski et al. [33]. with the cell wall composition of C. glutamicum. In the central metabolic pathway, the reconstructed model of $C$. glutamicum has pyruvate carboxylase in the anaplerotic pathway and lacks pyruvate formate lyase. In addition, $C$. glutamicum has 2 pathways for the biosynthesis of diaminopimelate, which is a precursor for lysine biosynthesis. One pathway involves the direct conversion of $\Delta 1$-piperideine-2,6-dicarboxylate to diaminopimelate, which is catalyzed by diaminopimelate dehydrogenase; the other pathway involves an indirect conversion, which is catalyzed by 4 independent enzymes [37]. Additionally, $C$. glutamicum does not have glycine C-acetyltransferase, which is involved in the conversion of threonine to glycine. These distinguishing characteristics of the metabolic pathways are necessary to represent the flux profile of $C$. glutamicum.

\section{Verification of the C. glutamicum genome-scale model: Measuring metabolic profiles under various oxygen supply conditions}

In order to verify the results of the FBA using our genomescale model of C. glutamicum, we compared the growth and metabolic profiles obtained by FBA simulations with those obtained by experiments performed at various oxygen levels. Here, we focused on the OUR as a parameter to change the metabolic profiles of C. glutamicum; this is because OUR is known to alter the metabolic profile drastically and is a key factor for controlling the productivity of several materials by this microorganism, such as organic acids. We performed a series of experiments involving the culture of C. glutamicum ATCC 13032 at various oxygen levels. The results of these experiments are summarized in Fig. 1(a). For aerobic conditions, i.e., conditions with relatively high OURs, we used continuous cultures with a dilution rate of $0.2 \mathrm{~h}^{-1}$; in these cultures, the oxygen supply was changed by altering the agitation speed to 800, 1000, and $1100 \mathrm{rpm}$ (experiments 3, 4, and 5 in Fig. 1(a)). Here, the steady state was defined as that $\mathrm{CV}$ of cell concentration measured by optical density $\left(\mathrm{OD}_{660}\right)$ is less than $10 \%$ over 10 hours. The production rates of $\mathrm{CO}_{2}$ and organic acids were measured in the steady states of the continuous cultures. We also confirmed that, for GUR, OUR, and production rates of organic acids, the ranges of deviation from the means were less than 5\% during the steady state. In microaerobic and oxygen anaerobic conditions, C. glutamicum exhibited low specific growth rate (e.g., less than $0.05 \mathrm{~h}^{-1}$ ), and maintaining a steady state in the continuous cultures was difficult. Thus, we used batch cultures in this study and altered the oxygen supply conditions during the culture. In the batch cultures, cells were first grown under aerobic conditions with aeration and a high agitation speed; during the exponential growth phase, nitrogen gas started to be supplied instead of air for maintaining the anaerobic condition (experiment 1) and the agitation speed was 
Table 2: Functional classification of metabolic reactions in a C. glutamicum genome-scale model.

\begin{tabular}{|c|c|c|c|}
\hline Carbohydrate metabolism & 45 & Metabolism of complex carbohydrate & 66 \\
\hline glycolysis/gluconeogenesis & 18 & arabinogalactan biosynthesis & 2 \\
\hline TCA cycle & 17 & dTDP-rhamnose biosynthesis & 5 \\
\hline pentose phosphate pathway & 8 & D-lactate metabolism & 5 \\
\hline Entner-Doudoroff pathway & 2 & GDP-mannose metabolism & 5 \\
\hline Energy metabolism & 17 & glycerol and glycerophosphodiester degradation & 2 \\
\hline Lipid metabolism & 32 & mevalonate pathway & 9 \\
\hline fatty acid biosynthesis & 15 & UDP-N-acetylgalactosamine biosynthesis & 7 \\
\hline phospholipid biosynthesis & 17 & UDP-glucose conversion & 3 \\
\hline Nucleotide metabolism & 84 & isopentenyl diphosphate biosynthesis & 8 \\
\hline PRPP biosynthesis & I & glutathione redox reactions & 4 \\
\hline purinebiosynthesis & 29 & myo-inositol biosynthesis & 2 \\
\hline pyrimidine biosynthesis & 23 & Polysaccharide biosynthesis & I \\
\hline nucleotide salvage pathway & 31 & peptidoglycan biosynthesis & 13 \\
\hline Amino acid metabolism & 103 & Metabolism of complex lipid & 13 \\
\hline glutamate biosynthesis & 2 & MAPc biosynthesis & I \\
\hline glutamine biosynthesis & 1 & PIM2 biosynthesis & 2 \\
\hline alanine biosynthesis & 3 & Mycolyl-ACP biosynthesis & I \\
\hline valine biosynthesis & 3 & polyamine biosynthesis & 7 \\
\hline aspartate biosynthesis & 1 & Corynomycolate biosynthesis & 2 \\
\hline lysine biosynthesis & 11 & Metabolism of cofactors and vitamins & 67 \\
\hline arginine biosynthesis & 9 & ATP maintenance & I \\
\hline asparagine biosynthesis & 2 & coenzyme $\mathrm{A}$ biosynthesis & 5 \\
\hline threonine biosynthesis & 2 & folate transformations & 2 \\
\hline isoleucine biosynthesis & 5 & formyITHF biosynthesis & 9 \\
\hline leucine biosynthesis & 4 & NAD biosynthesis & 19 \\
\hline proline biosynthesis & 5 & O-antigen biosynthesis & I \\
\hline serine biosynthesis & 3 & pantothenate biosynthesis & 6 \\
\hline tyrosine biosynthesis & 3 & riboflavin and FMN and FAD biosynthesis & 9 \\
\hline tryptophan biosynthesis & 6 & tetrahydrofolate biosynthesis & 15 \\
\hline cysteine biosynthesis & 2 & Metabolism of other amino acids & 9 \\
\hline phenylalanine biosynthesis & 3 & homoserine biosynthesis & I \\
\hline glycine biosynthesis & 1 & chorismate biosynthesis & 7 \\
\hline methionine biosynthesis & 17 & spermine biosynthesis & I \\
\hline histidine biosynthesis & 10 & Metabolism of sugars & 14 \\
\hline \multirow[t]{5}{*}{ interconversion of arginine, ornithine and proline } & 10 & trehalose biosynthesis & 2 \\
\hline & & starch biosynthesis & 12 \\
\hline & & Transport pathway & 31 \\
\hline & & Biomass synthesis & I \\
\hline & & Exchange pathway & 34 \\
\hline
\end{tabular}

The reaction "Biomass synthesis" indicates the hypothetical reaction to synthesize biomass, which represents the requirement of precursors and coenzymes for the biomass formation.

controlled to maintain a constant OUR for the microaerobic condition (experiment 2). After changing the oxygen supply conditions, the production rates of $\mathrm{CO}_{2}$ and organic acids were measured (the time series of cell concentration and organic acid concentrations in experiments 1 and 2 are presented in additional files 2 and 3 ). Here, we obtained GUR, OUR, and the production rate of organic acids by linear regression of the time series. To check the error of this regression, we also calculated the confidence intervals of the regression and found that the $95 \%$ confidence intervals are less than $10 \%$ from the mean. In experiment 1, data of OUR was set to zero since nitrogen gas was supplied to the fermentor instead of air. As shown in Fig. 1(a), the production rates of biomass, $\mathrm{CO}_{2}$, and organic acids depended on the oxygen supply conditions.
The experimental data presented in Fig. 1(a) indicated that under the anaerobic and micro-aerobic conditions (experiment 1 and 2), the cells converted most of the glucose to organic acids as lactate and succinate. With an increase in OUR/GUR ratio, cells changed their metabolism to produce acetate (experiment 3), and a further increase in OUR/GUR ratio resulted metabolic shift to $\mathrm{CO}_{2}$ production phase in which the tricarboxylic acid (TCA) cycle was activated (experiment 4 and 5).

Using the experimental data summarized in Fig. 1(a), we evaluated the results of the FBA simulations of our genome-scale model. In these simulations, we used biomass maximization as the objective function of FBA. We calculated the production yields of organic acids, $\mathrm{CO}_{2}$, 
and biomass using experimentally observed OUR and GUR values. The predicted yields are presented in Fig. 1(a). Also, in Fig. 1(b), we show a scatter plot of carbon yields, in which the carbon yields in the 5 sets of experimental and simulation results are presented. In Fig. 1(b), $\mathrm{x}$-axis shows the predicted yield by FBA simulation and $\mathrm{y}$ axis represents experimentally observed ones. As shown in the Fig. 1(a) and 1(b), the predictions of our genomescale model with maximization of the biomass yield agreed well with the experimentally obtained yields. For example, the FBA simulation predicted that $10 \%$ of carbon is secreted as acetate when OUR/GUR 1.5, which is consistent with the experimental result. The most significant discrepancy between the experimental results and FBA simulation was in the succinate production yield under the micro-aerobic and anaerobic conditions. The FBA simulation with biomass production maximization predicted that $10 \sim 20 \%$ of carbon is secreted as succinate in that condition, and the experimental results revealed that around $5 \%$ of carbon was secreted as succinate. The possible cause of this discrepancy will be discussed in the next section.

In order to evaluate the accuracy of the predictions by the FBA simulations in more details, we compared the results of the FBA simulations with the intracellular metabolic flux profile obtained by using a ${ }^{13} \mathrm{C}$-tracer experiment [10]. Here, we referred to the metabolic profile of $C$. glutamicum exponentially grown with glucose as the sole carbon source under aerobic conditions. The GUR and OUR were set to those measured experimentally. As presented in Fig. 2, the predicted flux for the pentose phosphate pathway (PPP) was quite similar to the experimentally obtained flux. However, in the anaplerotic and gluconeogenetic pathways, i.e., phosphoenolpyruvate to/from oxaloacetate, pyruvate to oxaloacetate, and malate to pyruvate, there seemed to be a discrepancy between the predicted and experimentally obtained results. This was because a cycle of metabolic flux in the anaplerotic and gluconeogenetic pathways does not affect the biomass production in the FBA simulation. Therefore, these fluxes were undetermined. However, when we compared the net fluxes from phosphoenolpyruvate/pyruvate to oxaloacetate/malate, which can be uniquely determined by biomass production maximization, the predicted and experimentally obtained fluxes were quite similar (19.5 in the FBA simulation and 18 in the experiment). Additionally, there was a discrepancy in the fluxes of the TCA cycle. The reason for the differences was clear, i.e., it was due to the differences in the precursor demands for the biomass production used in the ${ }^{13} \mathrm{C}$-tracer experiment and our simulation. For example, in our simulation result of aerobic condition, $14.7 \%$ of total carbon flux was consumed from acetyl-CoA to synthesis biomass components, such as lipid. In contrast, to obtain fluxes in the
${ }^{13} \mathrm{C}$-tracer experiment shown in Fig. 2, it was assumed that only $7.7 \%$ of total carbon flux was used for biomass synthesis from acetyl-CoA. This difference in the precursor demands resulted the discrepancy between simulation and experimental results in the fluxes in TCA cycle. It should be noted that after compensate the difference in the precursor demand, the fluxes in TCA cycle became more similar (data not shown).

\section{FBA of the metabolic profiles under various oxygen supply conditions}

Since the production yields of biomass, $\mathrm{CO}_{2}$, and organic acids agreed well between experimental results and FBA simulations, we further analyzed the changes in the intracellular metabolic profiles on changing the oxygen supply by using FBA simulations of our genome-scale model. In Fig. 3, the changes in the production yields are plotted as a function of OUR/GUR. As shown in the figure, the metabolic profiles can be classified into 5 phases. The schematic representations of the metabolic flux profiles of each phase are presented in additional file 4 . Phase I corresponds to the aerobic condition with enough oxygen supply; in this phase, the TCA cycle is activated. On decreasing the oxygen supply, the cells start to produce acetate (phase II). In this phase, the oxygen uptake is not enough to oxidize all the NADH produced when most glucose is converted to $\mathrm{CO}_{2}$ in the TCA cycle and the residual glucose is converted to acetate to produce ATP. Further decrease in the oxygen supply results in the production of lactate instead of acetate (phase III). In this phase, the carbon flux to the TCA cycle is almost stopped (except for fluxes to supply metabolites required for the production of biomass such as amino acids). The oxygen uptake is not enough to oxidize all the NADH produced in glycolysis; thus, lactate production is utilized as a mechanism to oxidize NADH in order to maintain the intracellular redox balance. In phase IV, succinate is produced instead of lactate. This is because the capacity of NADH oxidation is greater in the succinate production process than in the lactate production process. Two moles of NADH can be oxidized during the production of 1 mole of succinate from 1 mole of pyruvate, while only 1 mole of NADH can be oxidized during the production of 1 mole of lactate from 1 mole of pyruvate (see the profile (c) and (d) in additional file 4 for details of the metabolic profiles). For this reason, even though the ATP consumption is higher in the anaplerotic pathway than in the lactate production pathway, succinate production is preferred in order to maintain the intracellular redox balance under the microaerobic condition in phase IV. Further decrease in the oxygen supply results in an increase in the lactate production again (phase V). In this phase, with an increase in the lactate production, the metabolic flux from malate to pyruvate, which is catalyzed by malic enzyme (ME), is activated and the flux from glycolysis to the PPP decreases 
(a) experiment

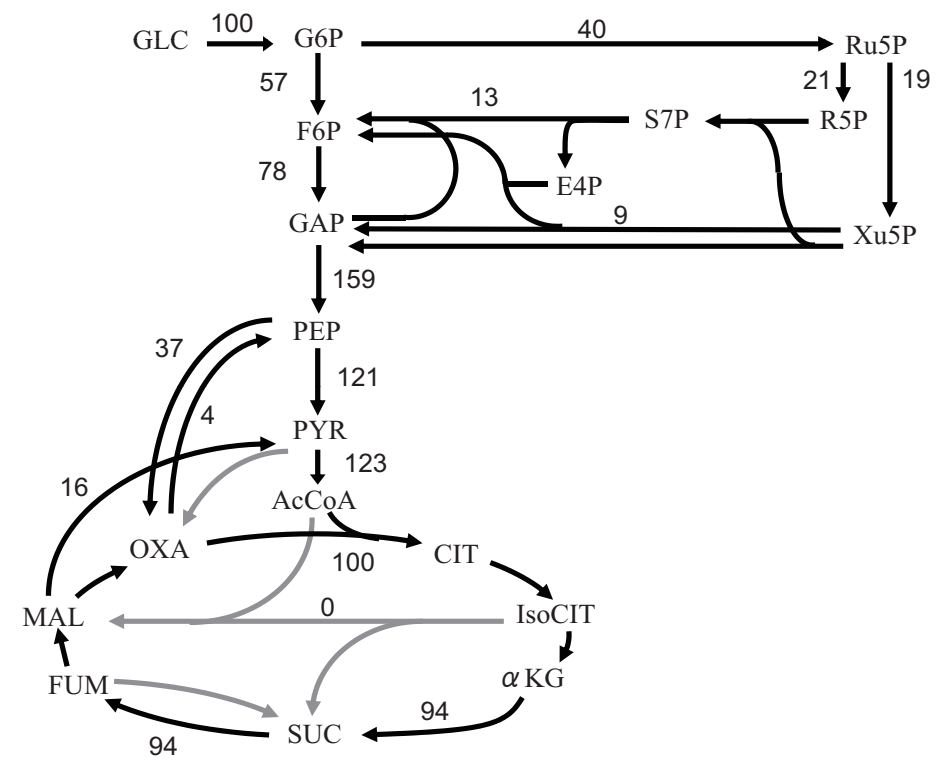

(b) simulation

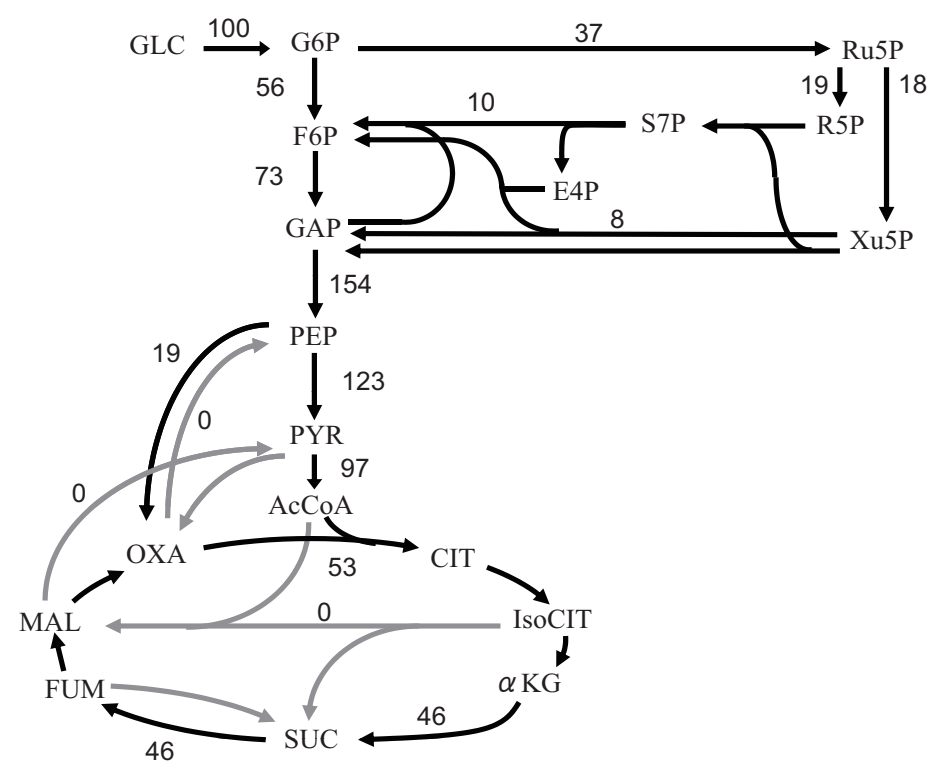

\section{Figure 2}

Metabolic flux profiles of $C$. glutamicum in the exponential growth phase. (a) The metabolic fluxes obtained by performing ${ }^{13} \mathrm{C}$-tracer experiment [10] and (b) the simulated metabolic fluxes with the same GUR and OUR are presented. The black and gray arrows represent reactions with non-zero and zero fluxes, respectively. Abbreviations are as follows: E4P, erythrose-4-phosphate; 6PG, 6-phospho-d-gluconate; Xu5P, xylulose-5-phosphate; Pyr, pyruvate; F6P, fructose-6-phosphate; GAP, glyceraldehyde-3-phosphate; R5P, ribose-5-phosphate; Ru5P, ribulose-5-phosphate; S7P, sedoheptulose-7-phosphate; G6P, glucose-6-phosphate; LYS, lysine; Suc, succinate; Cit, citrate; IsoCit, Isocitrate; aKG, a-ketoglutarate; MAL, malate; FUM, fumarate; OAA, oxaloacetate. 


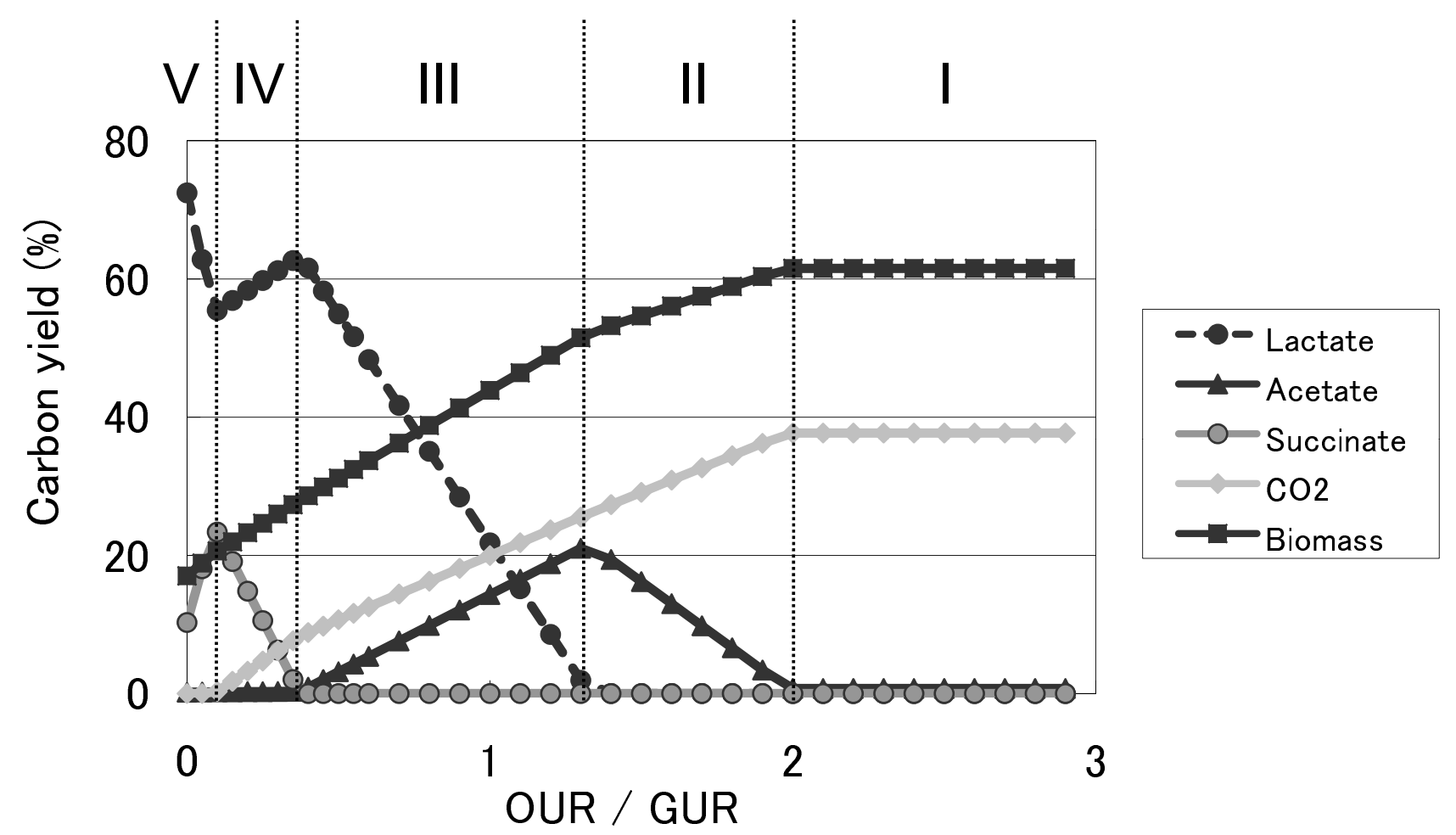

Figure 3

Result of FBA simulation: changes in the yields of organic acids, biomass, and carbon dioxide by changing the OUR/GUR ratio.

(see the profile (e) in additional file 4). Here, the flux for $\mathrm{ME}$ is enhanced in order to provide NADPH; the production of NADPH by ME allows a decrease in the flux to PPP, which is major supply source of NADPH in aerobic and microaerobic conditions. The decrease in the PPP flux results in an increase in the carbon fluxes for glycolysis and lactate production, which helps maintain the intracellular redox balance.

As shown in Fig. 1, the changes in the metabolic profiles in the FBA simulations mentioned above agreed well with the changes observed experimentally, except for the succinate production yield in the micro-aerobic and anaerobic conditions (phase IV and phase V). This discrepancy in the succinate production might be due to the differences between the simulation and experiment in the ATP demand for biomass production or in the $\mathrm{P} / \mathrm{O}$ ratio. As mentioned above, succinate production from pyruvate requires ATP consumption in the anaplerotic pathway; thus, the oxidation of NADH by such succinate production tends to be preferred when the ATP demand for bio- mass production is small or the $\mathrm{P} / \mathrm{O}$ ratio is large. In fact, when we changed the ATP demand for biomass production to $70.1 \mathrm{mmol}$ per $1 \mathrm{~g}$ biomass, which was 1.7 fold higher than the original coefficient based on the previous report, the predicted production yield of succinate in the micro-aerobic condition showed good agreement with the experimental one. However, this change of coefficient resulted in the decrease of the biomass yield, and a significant discrepancy in the biomass yield between simulation and experimental results arose, for example, it was more than $30 \%$ in aerobic condition (OUR/GUR > 2). This result might suggest that the coefficient for ATP in the genome-scale model should be changed depending on OUR.

\section{Comparison with previously proposed genome-scale model of C. glutamicum}

The difference between the previously proposed genomescale model of C. glutamicum [25] and our model is summarized in additional file 5 . It should be noted that the previous model could not represent the changes that 
occur in the metabolic profile on altering the oxygen supply. In the FBA simulation using that model, the metabolic profile was not altered by changing the oxygen supply condition. One reason for this discrepancy was the inclusion of inadequate reaction loops in the genomescale model. For example, that model consists of 2 transport reactions for urea; 1 reaction involves diffusion through the membrane without coupling to any other molecule and the other reaction is the urea-proton symport reaction. In FBA simulations, the combination of 2 such transport reactions results in an arbitrary proton flux from/to inside the cell; for example, the efflux of urea into the medium through the urea-proton symport and the intake of the same amount of urea by diffusion result in proton efflux without any changes in other metabolic balances except that of protons. Of course, the proton efflux can be balanced by proton-ATPase in the cell membrane with the generation of ATP. The genome-scale model reported in Ref. [25] consists several such inadequate reaction loops that allow the arbitrary generation of metabolites such as ATP and NADH; thus, representing the changes in the metabolic profiles by changing the oxygen supply condition is difficult. Furthermore, the model in Ref. [25] lacks the production pathways for lactate and succinate, which also make the representation of the metabolic state in the micro-aerobic condition difficult.

\section{Analysis of the metabolic profile in the amino acid production phase}

Since C. glutamicum is widely used for the industrial production of various amino acids such as lysine and glutamate, the prediction of metabolic profiles in the production phase of such amino acids is desirable for the improvement of the productivity. Thus, we validated the results of FBA simulation for lysine production by comparing the results with those of ${ }^{13} \mathrm{C}$-tracer experiment presented in Ref. [39]. In order to calculate the lysine production phase, we used lysine production rate instead of biomass production rate as the objective function of FBA to be maximized. The glucose uptake and biomass production rates were fixed at levels observed experimentally. The results revealed that the flux profile predicted by FBA agreed well with an experimentally obtained profile in a previous study, i.e., early production phase with exponential growth (Fig. five in Ref. [39]). These metabolic profiles are presented in Fig. 4. As shown in the figure, the maximal lysine production predicted by the FBA was similar to that obtained in the experiment. Additionally, a higher flux in the PPP in the experimental result $(69 \%)$ was consistent with that in the FBA simulation (69\%), and the net flux in the anaplerotic pathway also exhibited similar values ( $41 \%$ in both the experiment and the simulation). This clear consistency between the simulation and experimental results suggested that the experimentally obtained flux profile corresponds to that with highest lysine productivity under the condition of the observed growth rate. The most significant discrepancy between these 2 flux profiles was observed in the TCA cycle fluxes; this discrepancy was probably due to differences in the pyruvate and acetyl-CoA demands for the biomass production fluxes. Additionally, a discrepancy existed between the simulation result and the experimental result with regard to the use of 2 biosynthesis pathways for diaminopimelate, which is a precursor for lysine biosynthesis [37]. In the model simulation with the maximization of lysine production and fixed biomass production, the diaminopimelate dehydrogenase reaction was preferred; however, the experimental results indicated that both the pathways are active in the lysine production phase [38]. It should also be noted that the flux profile at the late exponential phase presented in Ref. [39] exhibited a larger discrepancy with the predicted flux profile obtained by our genome-scale model. This might be due to the difficulty in predicting a metabolic state at a lower growth rate by using FBA simulation, since there is a negative correlation between the growth rate and the volume of subspace corresponding metabolic state at the growth rate in the feasible flux space of FBA. That is, when the growth rate decreases, the range of possible metabolic states increases. Naturally, the difficulty in predicting the metabolic profile increases with a decrease in the cellular growth rate.

C. glutamicum is also widely used for glutamate production. However, the flux profile of the glutamate production phase cannot be represented by using the genomescale model so far. Glutamate production by C. glutamicum can be induced by several triggers such as biotin depletion [40], Tween 40 addition [41], and penicillin addition [42]. After receiving such a trigger, the cells cease growing and then start producing glutamate. Flux analysis using ${ }^{13} \mathrm{C}$-tracer experiments has revealed the changes in the metabolic flux profiles, indicating that a large fraction of carbon derived from glucose is converted to glutamate with the activation of the phosphoenol-pyruvate carboxylase pathway, which provides carbon flux to the TCA cycle [10]. However, the identification of a metabolic state in which glutamate is produced and cell growth is arrested is difficult using analysis by a genome-scale model; this is because the possible range of metabolic states is fairly large and no appropriate objective function exists to predict such a metabolic state. In order to represent the metabolic profile with glutamate production by simulating the genome-scale model, further improvements in the simulation scheme are necessary. For example, it is well known that a decrease in the 2-oxoglutarate dehydrogenase complex activity plays an essential role in the metabolic change in the glutamate production phase $[43,44]$. Additionally, a recent study suggested the existence of a membrane exporter of glutamate, whose conformation 
(a) experiment

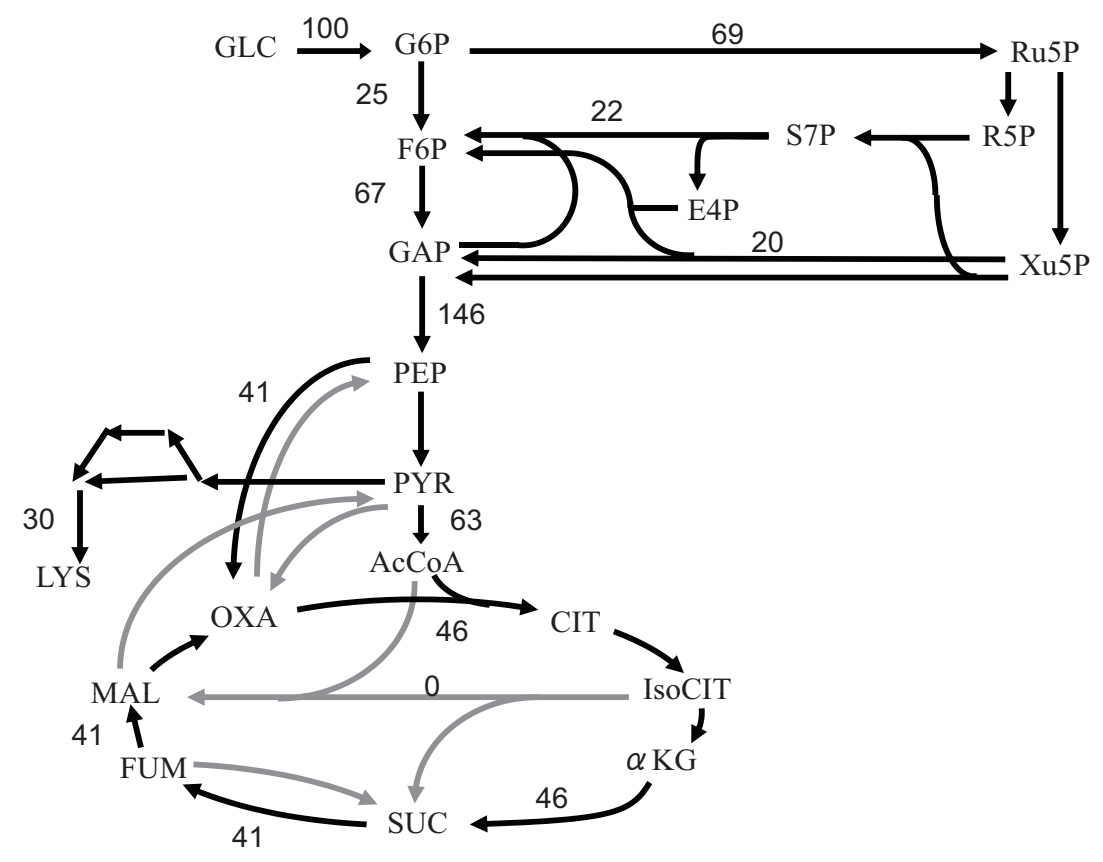

(b) simulation

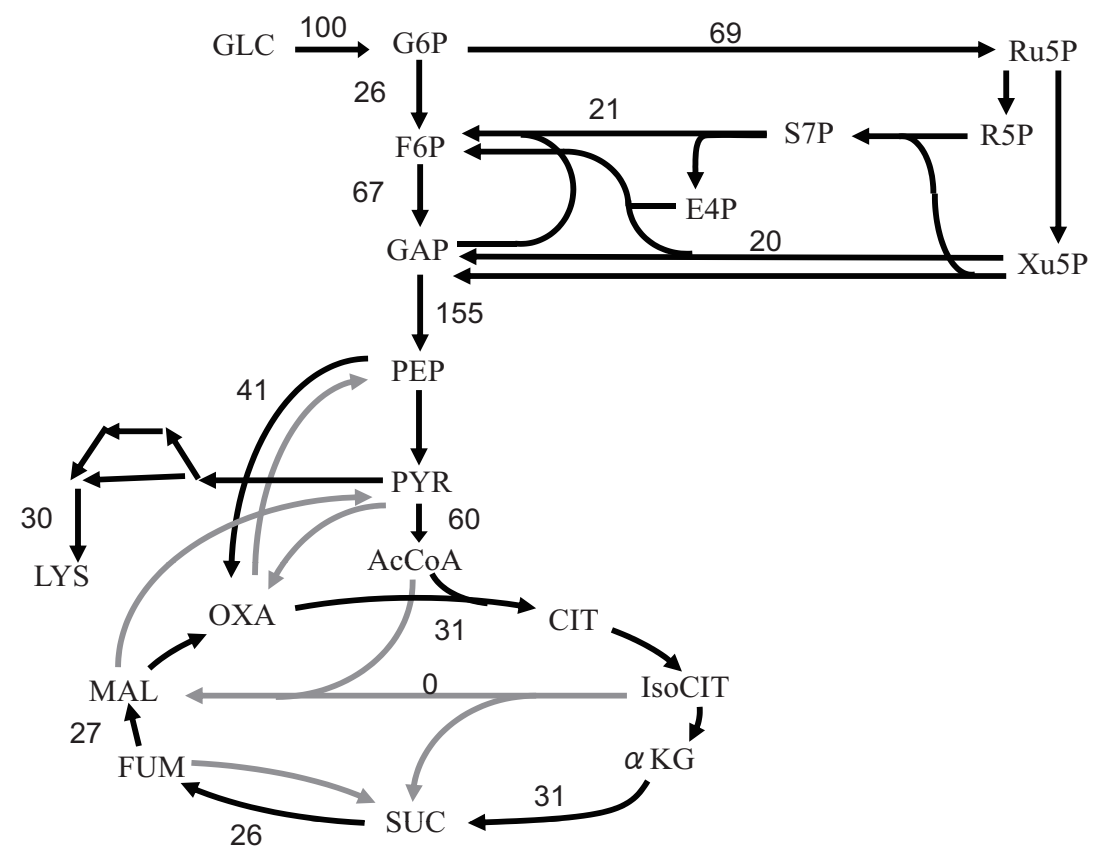

\section{Figure 4}

Metabolic flux profiles of $C$. glutamicum in the lysine production phase. (a) The metabolic profile obtained by performing the ${ }^{3} \mathrm{C}$-tracer experiment [38] and (b) the simulated metabolic profile obtained by using the same growth rate and by maximizing the lysine production rate are presented. 
change can be involved in the transition to the glutamate production phase [45]. The inclusion of such regulations of enzymatic activity might be important for the prediction of flux profiles of the glutamate production phase, and should be considered in future studies.

\section{Analysis of gene deletion for improvement in the production of organic acids}

Recently, the production of organic acids by C. glutamicum under oxygen deprivation conditions was proposed for increasing the productivity [6]. For the realization of this process, genetic engineering of $C$. glutamicum to improve organic acid productivity is desirable. Using FBA of our $C$. glutamicum metabolic model, we determined candidate genes for target deletion to improve the organic acid productivity under oxygen deprivation conditions. Table 3 shows the candidate pathways whose disruption by gene deletion resulted in an increased production of lactate or succinate. The FBA simulations with biomass production rate as the objective function were performed under the condition of OUR/GUR $=0.1$, in which the production rates of lactate and succinate are relatively high (See Fig. $3)$. For lactate overproduction, disruption of the succinate production pathway was important. Furthermore, the FBA simulation suggested that the disruption of reactions concerned with oxidative phosphorylation increased the lactate production rate. This was because of an increase in the demand of NADH oxidation caused by the disruption of oxidative phosphorylation, which resulted in an increase in the lactate production to oxidize NADH. Additionally, disrupting some reactions in the PPP resulted in an increase in the lactate production, which was due to decrease of NADPH synthesis in the PPP. Then, in order to compensate for NADPH production, which is required for cell growth, the reaction from malate to pyruvate, catalyzed by ME, was activated. The result of the FBA simula- tion indicated that the increased pyruvate was in turn converted to lactate. For succinate overproduction, disrupting only the lactate production pathway was effective in increasing the production rate of succinate. This result was natural since a large portion of the carbon flux is converted to lactate, as shown in the metabolic profile of the wild type. We further performed multiple gene deletion analysis to screen a set of genes to increase the lactate and succinate production rates; however, no further significant increase in the lactate and succinate production rates was observed (data not shown).

\section{Conclusion}

In this study, we developed a genome-scale metabolic model of C. glutamicum, which is commercially important for the production of amino acids and useful chemicals. This model includes hundreds of metabolites and reactions among them, and also includes that the hypothetical reaction representing synthesis of biomass to calculate the requirement of metabolites for cell growth. It should be stressed that, this model is not just a collection of all the public available data about the metabolic reaction of $\mathrm{C}$. glutamicum. Instead, we constructed the model by adding and deleting metabolic reactions to/from those on the databases, to make this model available for the flux balance analysis. For example, based on some literatures, we added some reactions to the metabolic model to fill gaps in the network to allow biomass formation. Also, we removed some reactions to avoid the formation of inadequate loop reactions, which makes impossible to calculate balance of metabolic fluxes due to the arbitrary generation of metabolites by this loop reaction as discussed above. Only after fixing these problems, we could use the metabolic model for FBA and predict the metabolic profiles. Using the genome-scale model, we performed FBA to understand the characteristics of the metabolic network

Table 3: Candidate reactions whose disruption increases the lactate or succinate production flux predicted by FBA simulations.

\begin{tabular}{|c|c|c|}
\hline Reaction disabled by gene deletion & Lactate production flux (mmol/gDW/h) & Growth rate $(\mathrm{I} / \mathrm{h})$ \\
\hline (Wild type) & $3.33(1.00)$ & $9.54 \times 10^{-2}(1.00)$ \\
\hline $\mathrm{ADP}+\mathrm{Pi}+4 \mathrm{H}[\mathrm{e}] \rightarrow \mathrm{ATP}+\mathrm{H}_{2} \mathrm{O}+\mathrm{H}$ & $5.13(1.54)$ & $8.50 \times 10^{-2}(0.89)$ \\
\hline $\mathrm{R} 5 \mathrm{P}+\mathrm{Xu} 5 \mathrm{P} \leftrightarrow \mathrm{S} 7 \mathrm{P}+\mathrm{GAP}$ & $5.02(\mathrm{I} .5 \mathrm{I})$ & $9.06 \times 10^{-2}(0.95)$ \\
\hline $\mathrm{MAL} \leftrightarrow \mathrm{FUM}+\mathrm{H}_{2} \mathrm{O}$ & $4.99(1.50)$ & $8.97 \times 10^{-2}(0.94)$ \\
\hline $\mathrm{G} 6 \mathrm{P}+\mathrm{NADP} \rightarrow 6 \mathrm{PGL}+\mathrm{NADPH}+\mathrm{H}$ & $4.99(1.50)$ & $9.06 \times 10^{-2}(0.95)$ \\
\hline Reaction disabled by gene deletion & Succinate production flux (mmol/gDW/h) & Growth rate $(1 / h)$ \\
\hline (Wild type) & $1.05(1.00)$ & $9.54 \times 10^{-2}(1.00)$ \\
\hline $\mathrm{NADH}+\mathrm{PYR}+\mathrm{H} \leftrightarrow \mathrm{LAC}+\mathrm{NAD}$ & $2.24(2.13)$ & $8.11 \times 10^{-2}(0.85)$ \\
\hline
\end{tabular}

Lactate and succinate production fluxes and growth rate of strains in which a reaction is disabled by gene deletion are presented. The production fluxes and growth rate were calculated with the parameters GUR $=0.3 \mathrm{mmol} / \mathrm{gDW} / \mathrm{h}$ and OUR $=0.03 \mathrm{mmol} / \mathrm{gDW} / \mathrm{h}$. Values in parenthesis represent fold change compared with those of wild type. Abbreviations are as follows: $\mathrm{H}$ [e], extracellular proton; MAL, malate; FUM, fumarate; G6P, glucose-6-phosphate; 6-PGL, 6-phospho-D-glucono-I,5-lactone; R5P, ribose-5-phosphate; Xu5P, xylulose-5-phosphate; S7P, sedoheptulose-7phosphate; GAP, glyceraldehyde-3-phosphate; PYR, Pyruvate; LAC, I-lactate. 
and to identify candidates of the target metabolic pathways that can be manipulated to improve organic acid production by C. glutamicum. The results revealed that the FBA agreed well with the experimental results; this suggests that when the cells grow exponentially, the metabolic profiles can be predicted by our genome-scale model with maximization of the biomass production rate. We also performed simulations to predict the metabolic profiles in amino acid production phases, and succeeded in representing the metabolic profiles in the lysine production phase. However, the glutamate production phase, in which the cells stop growing, could not be represented by the genome-scale model. Further improvements in the model, such as inclusion of some gene regulatory machinery, should be considered. Furthermore, we performed in silico screening to identify candidates of the target metabolic pathways that can be manipulated to improve organic acid production by C. glutamicum. Our results revealed that the disruption of $\mathrm{H}^{+}$-ATPase activity is the most effective in improving lactate production under oxygen deprivation conditions. In fact, recent studies showed that the disruption of $\mathrm{H}^{+}$-ATPase results in significant changes of metabolic flux profiles in C. glutamicum [46], although the flux profiles under oxygen deprivation have not yet been investigated. The experimental verification of this in silico screening remains as future works. We expect that further extensive studies using our genome-scale model with experimental verifications will enable us to understand in detail the characteristics of the metabolic networks of C. glutamicum.

\section{Competing interests}

The authors declare that they have no competing interests.

\section{Authors' contributions}

YS and MS performed the model construction and simulations. NS performed the culture experiments. CF participated in the design of the study and drafted the manuscript. TH rewrote the manuscript. HS conceived and supervised the study. All authors revised and approved the final manuscript.

\section{Additional material}

\section{Additional file 1}

List of metabolic reactions in the $\mathrm{C}$. glutamicum genome-scale model. The sheet "reactions" contains all the reactions in the $\mathrm{C}$. glutamicum genome-scale model categorized by their metabolic pathway and the sheet "abbreviated metabolites" enlists the abbreviations of the metabolites. Click here for file

[http://www.biomedcentral.com/content/supplementary/14752859-8-43-S1.xls]

\section{Additional file 2}

Growth and organic acid production by C. glutamicum under the anaerobic condition (experiment 1). The culture was initiated under aerobic conditions with air aeration and a high agitation speed, and $9 \mathrm{~h}$ after inoculation (indicated by a black arrow) the culture conditions were changed to no aeration and gentle agitation (100 rpm). The GUR, OUR, and production rates of organic acids, biomass, and carbon dioxide were calculated using the data obtained from 9 to $15 \mathrm{~h}$ after inoculation. Click here for file

[http://www.biomedcentral.com/content/supplementary/14752859-8-43-S2.pdf]

\section{Additional file 3}

Growth and organic acid production by $\mathrm{C}$. glutamicum under microaerobic conditions (experiment 2). The culture was initiated under aerobic conditions with air aeration and a high agitation speed, and $9 \mathrm{~h}$ after inoculation (indicated by a black arrow) the aeration rate was changed to $0.5 \mathrm{vm}$ and the OUR was maintained at a constant value $(0.5 \mathrm{mmol} /$ $g D W / h)$ by changing the agitation speed. The GUR, OUR, and production rates of organic acids, biomass, and carbon dioxide were calculated using the data obtained 9 to $15 \mathrm{~h}$ after inoculation.

Click here for file

[http://www.biomedcentral.com/content/supplementary/14752859-8-43-S3.pdf]

\section{Additional file 4}

Metabolic flux profiles of $\mathrm{C}$. glutamicum in the different phases. Figures (a) $\sim(e)$ show the schematic representations of the metabolic profiles in phases $I \sim V$, respectively. The thickness of the green arrows roughly corresponds to the metabolic fluxes; the gray arrows represent reactions with zero flux. The following abbreviations are used: $R e \_M q$, reduced form of menaquinone; $O x \_M q$, oxidized form of menaquinone.

Click here for file

[http://www.biomedcentral.com/content/supplementary/14752859-8-43-S4.pdf]

\section{Additional file 5}

Summary of differences between the genome-scale models of $C$. glutamicum in Ref.[25] and present study. The essential differences between the previous study [25] and our study are presented. Click here for file

[http://www.biomedcentral.com/content/supplementary/14752859-8-43-S5.pdf]

\section{Acknowledgements}

We thank to Mr. Satoshi Ohno for help of some simulations. This work was supported by Grants-in-Aid for Young Scientists (B) to CF and TH from the Ministry of Education, Culture, Sports, Science and Technology of Japan. This work was also supported in part by a grant from the Global COE (Center of Excellence) of the Ministry of Education, Culture, Sports, Science and Technology, Japan.

\section{References}

I. Kinoshita S, Udaka S, Shimono M: Studies on the amino acid fermentation. Appl Microbiol Jpn 1957, 3:193-205.

2. Udaka S: Screening method for microorganisms accumulating metabolites and its use in the isolation of micrococcus glutamicus. Jour Bacteriol 1960, 79(5):754-755.

3. Nakayama K, Kitada S, Kinoshita S: Studies on lysinefermentation. I. The control mechanism on lysine accumulation by homoserine and threonine. J Gen Appl Microbiol I96I, 7:145-I54. 
4. Kumagai $\mathrm{H}$ : Microbial Production of Amino Acids in Japan. Adv Biochem Eng Biotechnol 2000, 69:7I-85.

5. Leuchtenberger W, Huthmacher K, Drauz K: Biotechnological production of amino acids and derivatives: current status and prospects. Appl Microbiol Biotechnol 2005, 69(I): I-8.

6. Okino S, Inui M, Yukawa H: Production of organic acids by Corynebacterium glutamicum under oxygen deprivation. Appl Microbiol Biotechnol 2005, 68(4):475-480.

7. Inui M, Murakami S, Okino S, Kawaguchi H, Vertes AA, Yukawa $H$ : Metabolic analysis of Corynebacterium glutamicum during lactate and succinate productions under oxygen deprivation conditions. J Mol Microbiol Biotechnol 2004, 7(4): 182-196.

8. Stephanopoulos G: Metabolic fluxes and metabolic engineering. Metab Eng 1999, I (I): I-II.

9. de Graaf AA, Eggeling L, Sahm H: Metabolic engineering for Llysine production by Corynebacterium glutamicum. Adv Biochem Eng Biotechnol 2001, 73:9-29.

10. Shirai T, Fujimura K, Furusawa C, Nagahisa K, Shioya S, Shimizu H: Study on roles of anaplerotic pathways in glutamate overproduction of Corynebacterium glutamicum by metabolic flux analysis. Microb Cell Fact 2007, 6:19.

I1. Edwards JS, Palsson BO: The Escherichia coli MGI655 in silico metabolic genotype: its definition, characteristics, and capabilities. Proc Natl Acad Sci USA 2000, 97( ( 0):5528-5533.

12. Edwards JS, Ibarra RU, Palsson BO: In silico predictions of Escherichia coli metabolic capabilities are consistent with experimental data. Nat Biotechnol 200I, 19(2): I25-I30.

13. Feist AM, Scholten JC, Palsson BO, Brockman F], Ideker T: Modeling methanogenesis with a genome-scale metabolic reconstruction of Methanosarcina barkeri. Mol Syst Biol 2006, 2:

14. Feist AM, Henry CS, Reed JL, Krummenacker M, Joyce AR, Karp PD, Broadbelt LJ, Hatzimanikatis V, Palsson BO: A genome-scale metabolic reconstruction for Escherichia coli K-12 MG 1655 that accounts for 1260 ORFs and thermodynamic information. Mol Syst Biol 2007, 3:121.

15. Oh YK, Palsson BO, Park SM, Schilling CH, Mahadevan R: Genomescale reconstruction of metabolic network in Bacillus subtilis based on high-throughput phenotyping and gene essentiality data. J Biol Chem 2007, 282(39):2879|-28799.

16. Thiele I, Vo TD, Price ND, Palsson BO: Expanded metabolic reconstruction of Helicobacter pylori (iIT34 I GSM/GPR): an in silico genome-scale characterization of single- and doubledeletion mutants. J Bacteriol 2005, 187(16):5818-5830.

17. Oliveira AP, Nielsen J, Forster J: Modeling Lactococcus lactis using a genome-scale flux model. BMC Microbiol 2005, 5:39.

18. Duarte NC, Herrgard MJ, Palsson BO: Reconstruction and validation of Saccharomyces cerevisiae iND750, a fully compartmentalized genome-scale metabolic model. Genome Res 2004, I 4(7): 1298-1309.

19. Sheikh K, Forster J, Nielsen LK: Modeling hybridoma cell metabolism using a generic genome-scale metabolic model of Mus musculus. Biotechnol Prog 2005, 2 I (1): I I 2-12I.

20. Duarte NC, Becker SA, Jamshidi N, Thiele I, Mo ML, Vo TD, Srivas R, Palsson BO: Global reconstruction of the human metabolic network based on genomic and bibliomic data. Proc Natl Acad Sci USA 2007, 104(6): I 1777- 1782

2I. Ibarra RU, Edwards JS, Palsson BO: Escherichia coli K-I2 undergoes adaptive evolution to achieve in silico predicted optimal growth. Nature 2002, 420(69 / 2): $186-189$.

22. Fong SS, Palsson BO: Metabolic gene-deletion strains of Escherichia coli evolve to computationally predicted growth phenotypes. Nat Genet 2004, 36(10): I056-1058.

23. Lee SJ, Lee DY, Kim TY, Kim BH, Lee J, Lee SY: Metabolic engineering of Escherichia coli for enhanced production of succinic acid, based on genome comparison and in silico gene knockout simulation. Appl Environ Microbiol 2005, 7I(I 2):7880-7887.

24. Alper H, Miyaoku K, Stephanopoulos G: Construction of lycopene-overproducing $E$. coli strains by combining systematic and combinatorial gene knockout targets. Nat Biotechnol 2005, 23(5):612-616.

25. Kjeldsen KR, Nielsen J: In silico genome-scale reconstruction and validation of the Corynebacterium glutamicum metabolic network. Biotechnol Bioeng 2009, 102(2):583-597.

26. Karp PD, Ouzounis CA, Moore-Kochlacs C, Goldovsky L, Kaipa P, Ahren D, Tsoka S, Darzentas N, Kunin V, Lopez-Bigas N: Expansion of the BioCyc collection of pathway/genome databases to 160 genomes. Nucleic Acids Res 2005, 33(19):6083-6089.

27. Eggeling L, Bott M: Handbook of corynebacterium glutamicum. Boca Raton: CRC Press; 2005.

28. Burkovski A: Corynebacteria: Genomics and Molecular Biology. Norforlk: Caister Academic Press; 2008.

29. Birch HL, Alderwick LJ, Bhatt A, Rittmann D, Krumbach K, Sing A, Ba Y, Lowary TL, Eggeling L, Besra GS: Biosynthesis of mycobacterial arabinogalactan: identification of a novel $\alpha(I \rightarrow 3)$ arabinofuranosyltransferase. Molecular Microbiology 2008, 69(5): $1191-1206$.

30. Kacem R, De Sousa-D'Auria C, Tropis M, Chami M, Gounon P, LebIon G, Houssin C, Daffe M: Importance of mycoloyltransferases on the physiology of Corynebacterium glutamicum. Microbiology 2004, I 50(Pt I):73-84.

31. Cocaign-Bousquet M, Guyonvarch A, Lindley ND: Growth RateDependent Modulation of Carbon Flux through Central Metabolism and the Kinetic Consequences for Glucose-Limited Chemostat Cultures of Corynebacterium glutamicum. Appl Environ Microbiol 1996, 62(2):429-436.

32. Crick DC, Mahapatra S, Brennan PJ: Biosynthesis of the arabinogalactan-peptidoglycan complex of Mycobacterium tuberculosis. Glycobiology 200 I, I I(9): 107R-I I 8R.

33. Kalinowski J, Bathe B, Bartels D, Bischoff N, Bott M, Burkovski A, Dusch N, Eggeling L, Eikmanns BJ, Gaigalat L, et al.: The complete Corynebacterium glutamicum ATCC 13032 genome sequence and its impact on the production of L-aspartatederived amino acids and vitamins. J Biotechnol 2003, I04(I3):5-25.

34. Cocaign-Bousquet M, Lindley ND: Pyruvate overflow and carbon flux within the central metabolic pathways of Corynebacterium glutamicum during growth on lactate. Enzyme and Microbial Technology 1995, 17(3):260-267.

35. Shirai T, Nakato A, Izutani N, Nagahisa K, Shioya S, Kimura E, Kawarabayasi Y, Yamagishi A, Gojobori T, Shimizu H: Comparative study of flux redistribution of metabolic pathway in glutamate production by two coryneform bacteria. Metab Eng 2005, 7(2):59-69.

36. Ikeda $M$, Nakagawa S: The Corynebacterium glutamicum genome: features and impacts on biotechnological processes. Appl Microbiol Biotechnol 2003, 62(2-3):99-109.

37. Schrumpf B, Schwarzer A, Kalinowski J, Puhler A, Eggeling L, Sahm H: A functionally split pathway for lysine synthesis in Corynebacterium glutamicium. J Bacteriol I99I, I 73(14):45 10-4516.

38. Sonntag K, Eggeling L, De Graaf AA, Sahm H: Flux partitioning in the split pathway of lysine synthesis in Corynebacterium glutamicum. Quantification by I3C- and IH-NMR spectroscopy. Eur Jiochem 1993, 213(3): |325-1331.

39. Vallino JJ, Stephanopoulos G: Metabolic flux distributions in Corynebacterium glutamicum during growth and lysine overproduction. Reprinted from Biotechnology and Bioengineering, Vol. 4I, Pp 633-646 (1993). Biotechnol Bioeng 2000, 67(6):872-885

40. Shiio I, Otsuka SI, Takahashi M: Effect of biotin on the bacterial formation of glutamic acid. I. Glutamate formation and cellular premeability of amino acids. J Biochem 1962, 5 I:56-62.

4I. Takinami K, Yoshida H, Tsuri H, Okada H: Biochemical effects of fatty acid and its derivatives on L-glutamic acid fermentation. Part III. Biotin-Tween 60 relationship in the accumulation of L-glutamic acid and the growth of Brevibacterium lactofermentum. Agric Biol Chem 1965, 29:35I-359.

42. Nunheimer TD, Birnbaum J, Ihnen ED, Demain AL: Product inhibition of the fermentative formation of glutamic acid. Appl Microbiol 1970, 20(2):215-217.

43. Shimizu H, Tanaka H, Nakato A, Nagahisa K, Kimura E, Shioya S: Effects of the changes in enzyme activities on metabolic flux redistribution around the 2-oxoglutarate branch in glutamate production by Corynebacterium glutamicum. Bioprocess Biosyst Eng 2003, 25(5):291-298.

44. Bott M: Offering surprises: TCA cycle regulation in Corynebacterium glutamicum. Trends Microbiol 2007, 15(9):417-425.

45. Nakamura J, Hirano S, Ito H, Wachi M: Mutations of the Corynebacterium glutamicum NCgll 221 gene, encoding a mechanosensitive channel homolog, induce L-glutamic acid production. Appl Environ Microbiol 2007, 73( I4):449| -4498. 
46. Aoki R, Wada M, Takesue N, Tanaka K, Yokota A: Enhanced glutamic acid production by a $\mathbf{H + - A T P a s e - d e f e c t i v e ~ m u t a n t ~}$ of Corynebacterium glutamicum. Biosci Biotechnol Biochem 2005, 69(8): | $466-\mid 472$.

Publish with Biomed Central and every scientist can read your work free of charge

"BioMed Central will be the most significant development for disseminating the results of biomedical research in our lifetime. " Sir Paul Nurse, Cancer Research UK

Your research papers will be:

- available free of charge to the entire biomedical community

- peer reviewed and published immediately upon acceptance

- cited in PubMed and archived on PubMed Central

- yours - you keep the copyright

Submit your manuscript here:

http://www.biomedcentral.com/info/publishing_adv.asp
BioMedcentral 